\title{
PROPERTIES OF CONFLICT SETS IN THE PLANE
}

\author{
DIRK SIERSMA \\ Mathematisch Instituut \\ Universiteit Utrecht \\ Postbus 80.010, 3508 TA Utrecht, The Netherlands \\ E-mail: siersma@math.uu.nl
}

\begin{abstract}
This paper studies the smoothness and the curvature of conflict sets of the distance function in the plane. Conflict sets are also well known as 'bisectors'. We prove smoothness in the case of two convex sets and give a formula for the curvature. We generalize moreover to weighted distance functions, the so-called Johnson-Mehl model.
\end{abstract}

1. Introduction. We consider two regions in the Euclidean plane. A classical problem is to study the set of points of equal distance to the two sets. If the sets are points, lines or circles we get in this way parabolas, ellipses, hyperbolas and lines.

The sets of equal distance are known under several names: bisectors (in computational geometry), equi-distance lines, conflict lines of the distance function (in singularity theory), cut locus, etc.

Definition 1. Let $d$ denote the Euclidean distance function; $\mathbf{A}$ and $\mathbf{B}$ two closed sets in the plane $\mathcal{E}$. By $d(x, \mathbf{A})$ we denote the distance from a point $x$ to $\mathbf{A}$.

$$
\begin{aligned}
\operatorname{Conf}(\mathbf{A}, \mathbf{B})=\{x \in \mathcal{E} \mid d(x, \mathbf{A})=d(x, \mathbf{B})\} & \text { Conflict set, } \\
\operatorname{Terr}(\mathbf{A}, \mathbf{B})=\{x \in \mathcal{E} \mid d(x, \mathbf{A})<d(x, \mathbf{B})\} & \text { Territory of } \mathbf{A} \text { w.r.t. } \mathbf{B}, \\
\operatorname{Terr}(\mathbf{B}, \mathbf{A})=\{x \in \mathcal{E} \mid d(x, \mathbf{A})>d(x, \mathbf{B})\} & \text { Territory of } \mathbf{B} \text { w.r.t. } \mathbf{A} .
\end{aligned}
$$

We can also start from iso-distance lines (sets) with respect to $\mathbf{A}$ and $\mathbf{B}$. The conflict sets are precisely the intersections between iso-distance lines with the same distance. We can imagine the conflict set as the places where wave fronts from $\mathbf{A}$ and $\mathbf{B}$ meet at the first time.

Moreover one can consider circular discs, which are tangent to both $\mathbf{A}$ and $\mathbf{B}$. The centers of the discs are exactly the points on the conflict set. If one considers not discs, but only circles, tangent to both sets one finds in general a bigger locus of centers, since

1991 Mathematics Subject Classification: Primary 53A04; Secondary 68 U05.

The paper is in final form and no version of it will be published elsewhere. 
then the tangency is also allowed from the 'inside' of the circle. The methods of this paper apply with appropriate changes also to this more general type of bisector.

Below we study first the smoothness of the conflict set. Without differentiability assumptions about the boundaries this is not always the case, but if the boundaries are convex there is well defined tangent line. Next we give a formula for the curvature in a point $P$ of the conflict set. We use as data the distance to the sets, the angle between the projection lines and the curvatures at the projections of $P$.

Next we generalize this to weighted distances, and also here we show differentiability in the convex case and give a formula for the curvature.

The methods of this paper are elementary. We use planar geometry and squeezing arguments for the smoothness and elementary differential geometry for the curvature formulas.

Conflict sets play an important role in computational geometry (robotics, etc.). Moreover they have already been thoroughly studied in the history, where ellipses, parabolas, hyperbolas occurred in this context. This paper relates this old and important part of geometry with the recent investigations of conflicts sets in the plane.

While preparing this paper we profited from discussions with Aad Goddijn, who supplied the elementary proof of Theorem 1 . This was the starting point of the paper. We restrict in this paper to curves in the plane. Generalizations to higher dimensions are work in progress, we refer to $[\mathrm{SSG}]$ and $[\mathrm{Ma}]$.

2. Smoothness. In several sources the smoothness of the conflict set is mentioned or supposed in the case of convex sets $\mathbf{A}$ and $\mathbf{B}$. In non-convex cases the conflict set is not always smooth. In a certain sense the smoothness is remarkable since usual differential topology gives for $C^{k}$-boundaries a $C^{k-1}$-conflict set. Although the smoothness in the general convex case without differentiability conditions seems to be 'folklore', we could not find a complete proof in the literature. Goddijn [Go] supplied the following elementary proof, which gives also information about the position of the tangent lines. Goddijn's arguments also apply to the differentiability of iso-distance sets and generalize easily to higher dimensions and to several situations in the non-convex cases.
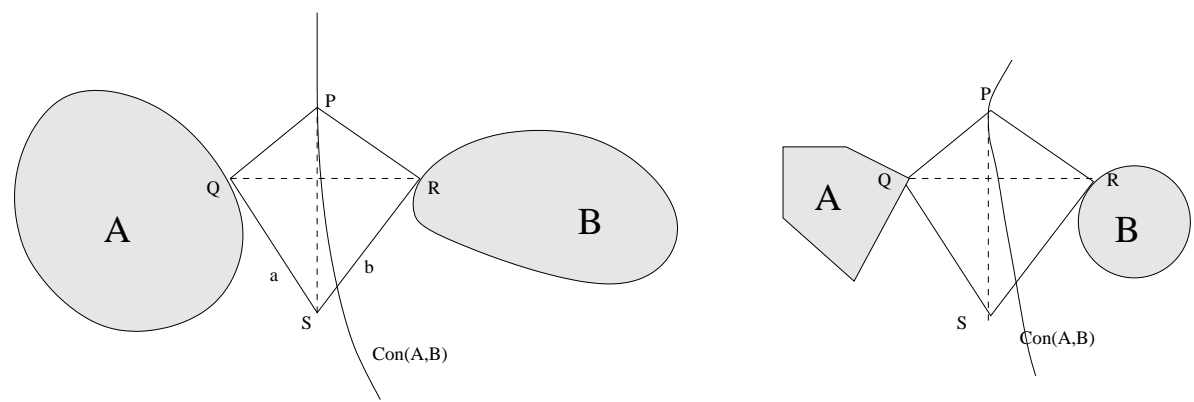

Figure 1. The conflict set and the kite of a point $P$ on it

Definition 2. The kite construction (Figure 1 ). Let $P$ be a point on $\operatorname{Conf}(\mathbf{A}, \mathbf{B})$. Consider the projections $Q$ of $P$ onto $\mathbf{A}$, and $R$ of $P$ onto $\mathbf{B}$. Let $a$ be the line through $Q$ which is perpendicular to $P Q$ and $b$ be the line through $Q$ which is perpendicular to $P R$. 
Let $S$ be the intersection point of $a$ and $b$. The quadrangle $P Q S R$ is a kite, the kite associated to the point $P$ on the conflict set. We call $P S$ the kite-axis. It is the bisector between the two projection lines $P Q$ and $P R$.

In case $a$ and $b$ are parallel, we have no finite intersection point, but we assume the intersection point at infinity. In this analogy we call the line through $P$ which is parallel to both $a$ and $b$ the axis of the 'kite'.

TheOREM 1. If $\mathbf{A}$ and $\mathbf{B}$ are convex sets in the Euclidean plane, then their conflict set is differentiable. Moreover the tangent line is the axis of the associated kite, which is also the bisector between the projection lines from the corresponding point.

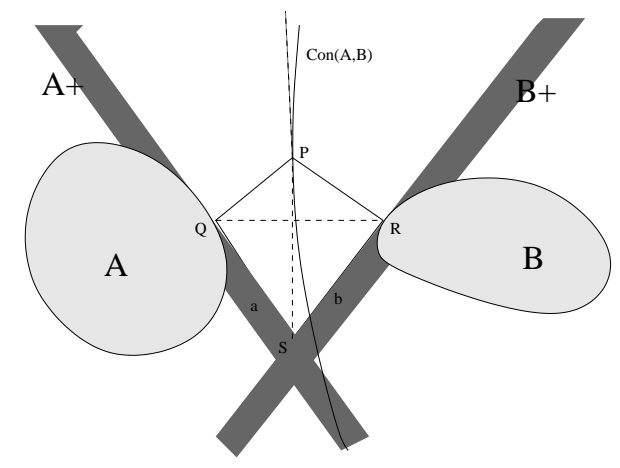

Figure 2. The construction of $\mathbf{A}^{+}$and $\mathbf{B}^{+}$

Proof. Let $P$ be a point on $\operatorname{Conf}(\mathbf{A}, \mathbf{B})$. Consider the associated kite $P Q S R$. Let $\mathbf{A}^{+}$be the closed half plane, which contains the region $\mathbf{A}$, and is bounded by the line $a$ (here convexity is used). Let $\mathbf{B}^{+}$be defined similarly. We continue now by increasing $\mathbf{A}$ to $\mathbf{A}^{+}$, decreasing $\mathbf{B}$ to $R$, doing the same in the other way and comparing their territories (Figure 2). For an arbitrary point $x$ on $\operatorname{Conf}(\mathbf{A}, \mathbf{B})$ we have

$$
\begin{aligned}
d\left(x, A^{+}\right) \leq d(x, \mathbf{A}) & =d(x, \mathbf{B}) \leq d(x, R), \\
d(x, Q) \geq d(x, \mathbf{A}) & =d(x, \mathbf{B}) \geq d\left(x, B^{+}\right) .
\end{aligned}
$$

From this it follows that $x \in \operatorname{Terr}\left(\mathbf{A}^{+}, R\right)$ and $x \in \operatorname{Terr}\left(\mathbf{B}^{+}, Q\right)$. Both territories are bounded by parabolas, which have only one point in common, namely $P$ (Figure 3 ).

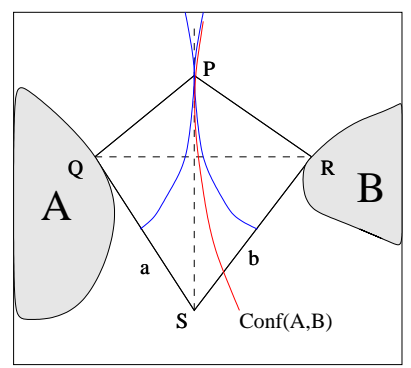

Figure 3. The squeezing 
The conflict set $\operatorname{Conf}(\mathbf{A}, \mathbf{B})$ is included in the closed 'sector' defined by (the closure of) the complements of these territories. The two parabolas have at $P$ the same tangent, which is also the tangent of the conflict set. We can also consider the bisector between $a$ and $b$ as part of $\operatorname{Conf}\left(\mathbf{A}^{+}, \mathbf{B}^{+}\right)$, which is the axis of the kite. Also this conflict set is squeezed between $\mathbf{A}^{+}$and $\mathbf{B}^{+}$, and is therefore the tangent line in $P$ to both parabolas.

The kite-property mentioned above is well known in the classical cases parabola (point-line); ellipse (circle, point inside); hyperbola (circle, point outside). It is the socalled tangent property, which can be used to construct the tangent line to a point on the conic. Moreover this is directly related to the reflection properties (Figure 4).
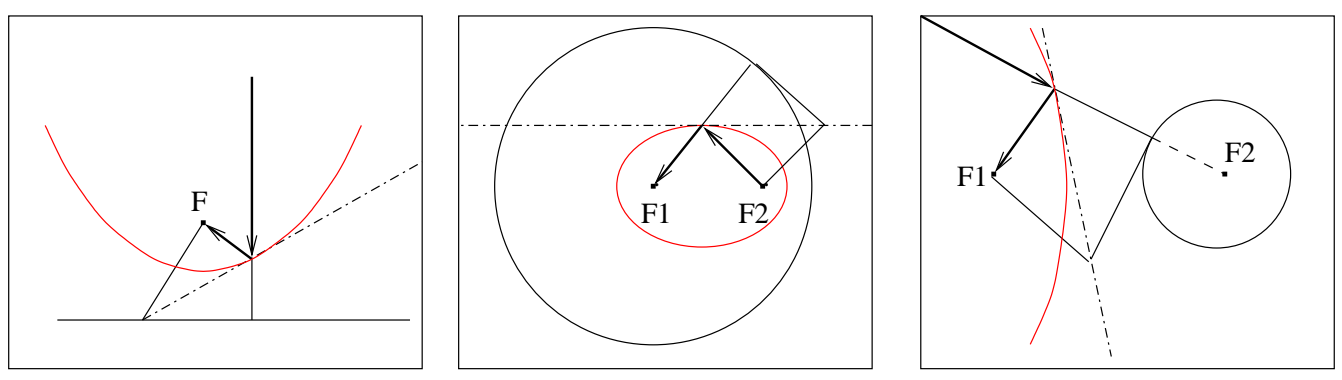

Figure 4. Tangent and reflection properties of a conic

Remark 1 . The locus of all points $P$ of the kite defines the conflict set. But there seems to be an interesting duality. One can also consider the locus of the points $S$, the base point of the kite. This set can be described as the locus of points where there exist tangent lines to $\mathbf{A}$ and $\mathbf{B}$ with the same length. It is quite possible that this set has been studied before. Also the case of tangency to the same set in two different points is an interesting subject.

3. Curvature. First we discuss the curvature of a iso-distance set to a region in the plane. We assume the boundary $C^{2}$ and also the iso-distance set is now at least $C^{2}$-smooth for small distance. More precisely: the iso-distance set is nonsingular and $C^{2}$ at points which are not on the evolute (or focal set) of the boundary [Mi]. We also refer to $[\mathrm{Be}],[\mathrm{BG}]$ and $[\mathrm{Po}]$ for background about conic sections and differential geometry.

Remark that this condition is sufficient to define curvature on smooth points of the isodistance sets. Examples show that with a lower degree of differentiability of the boundary curvature can be also defined in lots of points in several other situations, such as 'corners', where the iso-distance sets become arcs of circles.

Proposition 2. Let $P^{*}$ a point on the iso-r-distance set and $P$ the corresponding foot. Let $\kappa$ be the curvature and $\rho$ be the radius of curvature of the boundary curve in $P$ and let $\kappa^{*}$, resp. $\rho^{*}$, be the corresponding notions in $P^{*}$ for the iso-distance curve.

Then

$$
\rho^{*}=\rho+r \quad \text { and } \quad \kappa^{*}=\frac{\kappa}{1+\kappa r} .
$$


Proof. The statement about the $\rho$ follows from the fact that $\rho$ is the radius of the best tangential circle.

In the case $\rho=-r$ one has a point of the evolute, where the iso-distance set is not differentiable. In case of a corner $\rho^{*}=r$.

Proposition 3. Curvature of conflict sets in the plane.

Let $\mathbf{A}_{1}$ and $\mathbf{A}_{2}$ be two disjoint convex sets in the plane with $C^{3}$ boundaries. Then

$$
\kappa=\frac{1}{2}\left(\frac{\kappa_{1}}{1+\kappa_{1} r}-\frac{\kappa_{2}}{1+\kappa_{2} r}\right) \sin \phi .
$$

Here $\kappa$ is the curvature in a point $P$ of the conflict set, $\kappa_{i}$ are the curvatures in the feet of $P$ on the boundary of $\mathbf{A}_{i}$, and $r$ is the distance from $P$ to $\mathbf{A}_{i}$.

Proof. We use the kite-construction (cf. Figure 5) in order to determine the normal vector to the conflict set.

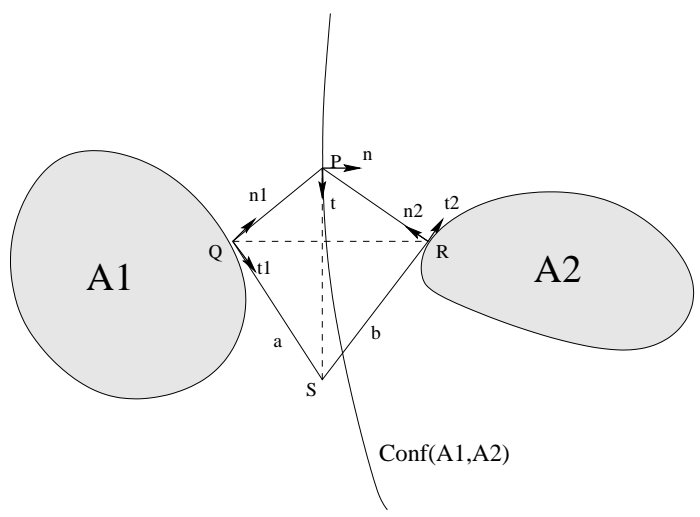

Figure 5. Definitions of Frenet-Serret frames

We use the notation: $\mathbf{n}, \mathbf{n}_{1}, \mathbf{n}_{2}$ are the unit-normal vectors, respectively to the conflict set, the boundary of $\mathbf{A}_{1}$ and $\mathbf{A}_{2} ; \mathbf{t}, \mathbf{t}_{1}, \mathbf{t}_{2}$ are the unit-tangents, respectively to the conflict set, the boundary of $\mathbf{A}_{1}$ and $\mathbf{A}_{2}$. Also $\phi$ is the angle between the vectors $\mathbf{n}_{i}$ and $\mathbf{t}$.

We have:

$$
\mathbf{n}=\frac{\mathbf{n}_{1}-\mathbf{n}_{2}}{2 \sin \phi} \text { and } \mathbf{t}=-\frac{\mathbf{n}_{1}+\mathbf{n}_{2}}{2 \cos \phi} .
$$

We replace $\mathbf{A}_{1}$ and $\mathbf{A}_{2}$ by the $r$-distance sets. The boundaries intersect at $P$. This does not influence the conflict set and the tangent and normal vectors.

Let $s$ be arc length for the conflict set and $s_{i}$ for the boundaries of the $r$-distance sets.

$$
\begin{aligned}
\frac{d \mathbf{n}}{d s}=\left(\frac{d \mathbf{n}_{1}}{d s_{1}} \cdot \frac{d s_{1}}{d s}-\frac{d \mathbf{n}_{2}}{d s_{2}} \cdot \frac{d s_{2}}{d s}\right) & \frac{1}{2 \sin \phi}+\left(\mathbf{n}_{1}-\mathbf{n}_{2}\right) \cdot(\ldots) \\
& =\left(\kappa_{1}^{*} \mathbf{t}_{1} \frac{d s_{1}}{d s}-\kappa_{2}^{*} \mathbf{t}_{2} \frac{d s_{2}}{d s}\right) \frac{1}{2 \sin \phi}+\left(\mathbf{n}_{1}-\mathbf{n}_{2}\right) \cdot(\ldots) .
\end{aligned}
$$

In order to compute the curvature $\kappa$ it is now sufficient to take the inner product with $\mathbf{t}$. Note that

$$
\mathbf{t}_{1} \bullet \mathbf{n}_{2}=\sin 2 \phi=\mathbf{t}_{2} \bullet \mathbf{n}_{1} ; \quad\left(\mathbf{n}_{1}-\mathbf{n}_{2}\right) \bullet \mathbf{t}=0
$$


and moreover

$$
\frac{d s_{1}}{d s}=\frac{d s_{2}}{d s}=\sin \phi
$$

So we get

$$
\begin{aligned}
\kappa=\left(\kappa_{1}^{*} \mathbf{t}_{1} \bullet \mathbf{n}_{2} \frac{d s_{1}}{d s}-\kappa_{2}^{*} \mathbf{t}_{2} \bullet \mathbf{n}_{1} \frac{d s_{2}}{d s}\right) \frac{1}{4 \sin \phi \cdot \cos \phi} \\
=\frac{1}{2}\left(\kappa_{1}^{*} \frac{d s_{1}}{d s}-\kappa_{2}^{*} \frac{d s_{2}}{d s}\right)=\frac{1}{2}\left(\kappa_{1}^{*}-\kappa_{2}^{*}\right) \sin \phi .
\end{aligned}
$$

This completes the proof.

Remark 2. For a usual hyperbola, considered as the conflict set between a point and a disc of radius $c$, we get the following formula:

$$
\kappa=\frac{1}{2}\left(\frac{1}{r}-\frac{1}{r+c}\right) \sin \phi .
$$

4. Weighted distances, the Johnson-Mehl model. In this model we give weights $a$ and $b$ to each of the sets $\mathbf{A}$ and $\mathbf{B}$. The new distances are defined by

$$
d_{w}(x, A)=\frac{1}{a} d(x, \mathbf{A})
$$

where $d(x, \mathbf{A})$ is the Euclidean distance.

In the weighted metric the conflict set is given by

$$
\operatorname{Conf}_{a: b}(\mathbf{A}, \mathbf{B})=\frac{d(x, \mathbf{A})}{a}=\frac{d(x, \mathbf{B})}{b} .
$$

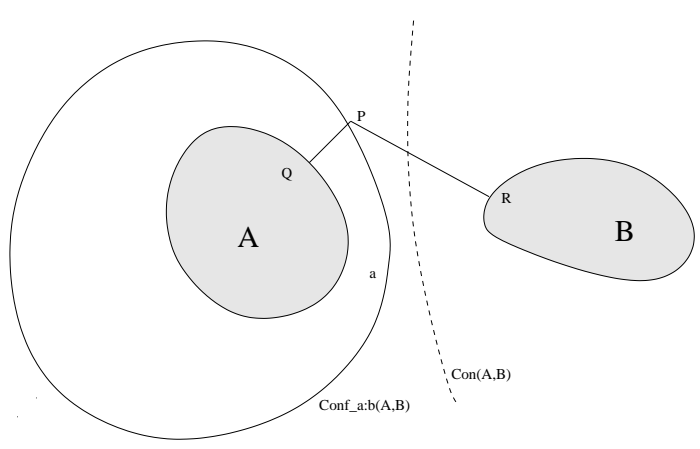

Figure 6. Weighted conflict sets

For $\mathbf{A}$ and $\mathbf{B}$ compact sets the weighted conflict set is a compact curve if $a \neq b$. Only in the case $a=b$ is the conflict set non-bounded (Figure 6).

We list (cf. Figure 7) the JM-conflict sets in the following cases (we assume $a \neq b$ ):

- point-point: The JM-bisector is a circle (the circle of Apollonius).

- line-line: The JM-bisector is a pair of straight lines.

- line-point: The JM-parabola is an (Euclidean) ellipse if $a>b$ or a hyperbola if $a<b$. 

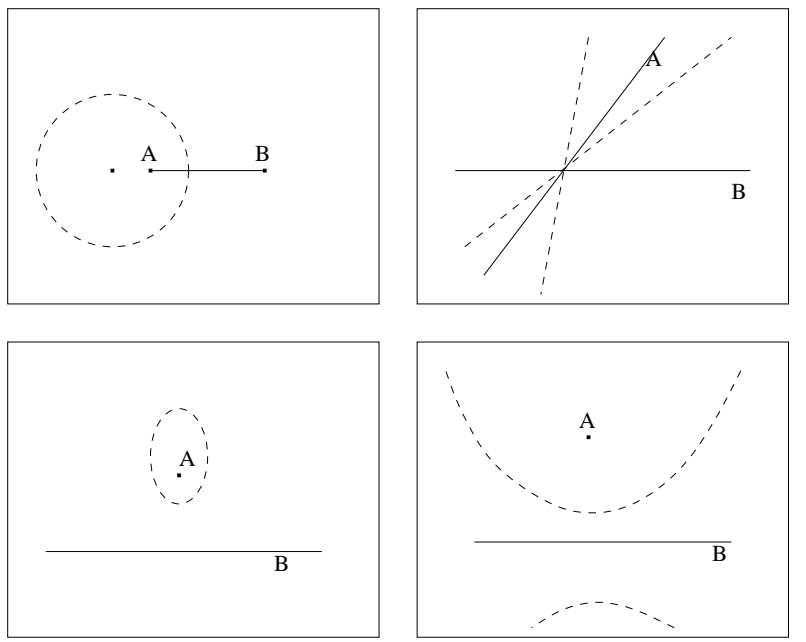

Figure 7. Weighted conflict sets

In fact these give one of the possible definitions for a conic section. According to Coxeter [Cox] older definitions for a conic, proposed by Menaechmus about 340 B.C. are reconciled with this one by Pappus of Alexandria (fourth century), or possibly by Euclid.

We next repeat the 'kite'-construction in the weighted case. This produces for every point $P$ on $\operatorname{Conf}_{a: b}(\mathbf{A}, \mathbf{B})$ a quadrangle $P Q S R$. This JM-kite has a circumscribed circle. In Figure 8 we show two different positions of the JM-kite.
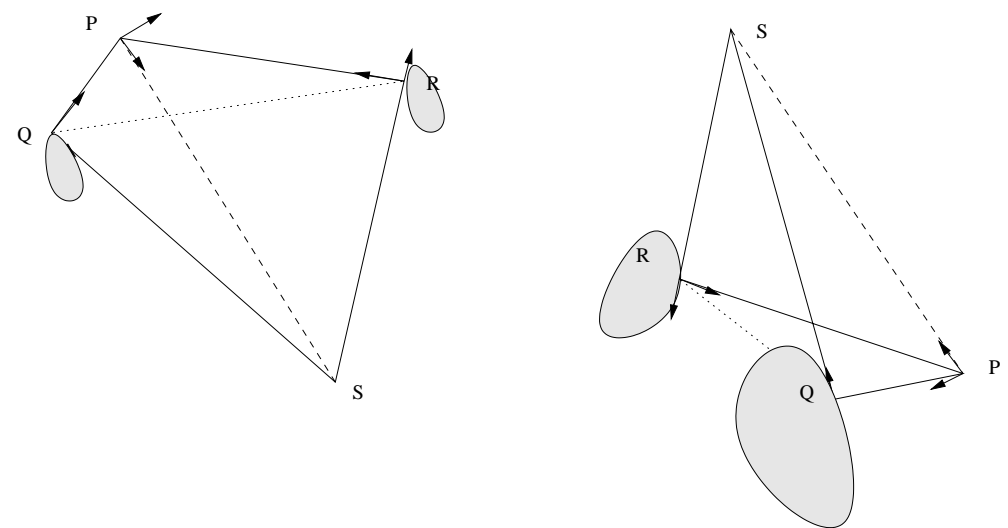

Figure 8. Two JM-kites

Geometrical formulas about the JM-kite. The shape of the JM-kite is determined by the ratio $a: b$ and the top angle $\gamma=\angle Q P R$. These data determine $\alpha=\angle Q P S$ and $\beta=\angle R P S$ :

where

$$
\sin \alpha=\frac{b-a \cos \gamma}{c}, \quad \sin \beta=\frac{a-b \cos \gamma}{c}
$$

$$
c=\sqrt{a^{2}+b^{2}-2 a b \cos (\alpha+\beta)} .
$$


This recalls to the cosine rule in triangle $Q P R$. The length of the sides and diagonals of the JM-kite are proportional with the JM-distance $r$. We have the formulas:

$$
\begin{array}{ll}
P Q=r a, & P R=r b, \\
Q S=\frac{b-a \cos \gamma}{\sin \gamma} r, & R S=\frac{a-b \cos \gamma}{\sin \gamma} r, \\
Q R=r c, & P S=r \frac{c}{\sin \gamma} .
\end{array}
$$

TheOrem 4. If $\mathbf{A}$ and $\mathbf{B}$ are convex sets in the Euclidean plane, then their conflict set is differentiable. Moreover the tangent line is the axis of the associated JM-kite, which is also the JM-bisector between the projection lines from the corresponding point.

Proof. This is similar to the Euclidean case. The conflict set is squeezed between $\operatorname{Terr}\left(A^{+}, R\right)$ and $\operatorname{Terr}\left(B^{+}, Q\right)$ (Figure 9). Also the diagonal $P S$ is in the complement.

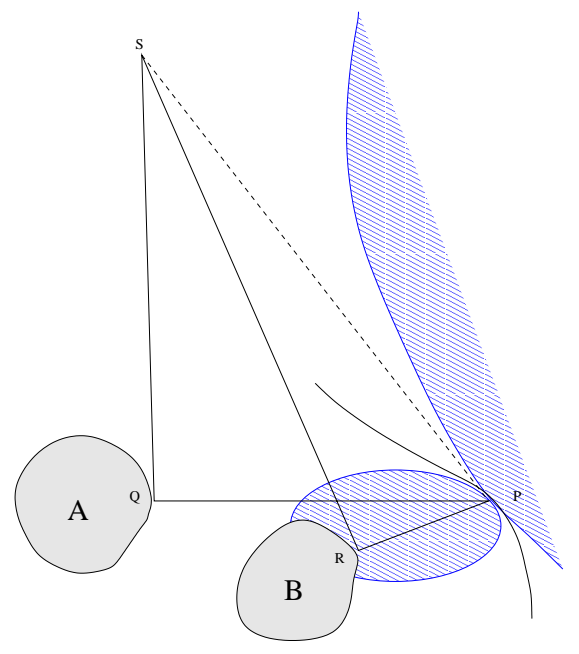

Figure 9. Weighted squeezing

Remark 3. In case $\mathbf{A}$ and $\mathbf{B}$ are points $A$ and $B$ the tangent property has the following geometric meaning:

Given a triangle $A B P$. Let the line through $A$ perpendicular to $A P$ and the line through $B$ perpendicular to $B P$ intersect each other in $S$. Let $K$ and $L$ be the intersection points of the outer and inner bisectors of angle $A P B$ with the line $A B$. Then $P S$ is tangent to the circle with $K L$ as diameter (Figure 10).

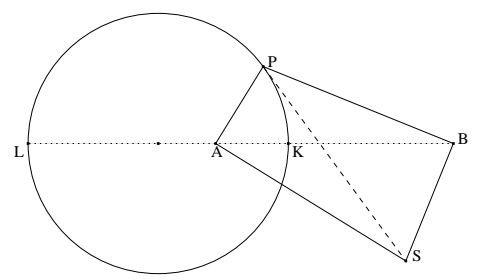

Figure 10. Illustration of Remark 3 
Proposition 5. Curvature of JM-conflict sets in the plane.

Let $\mathbf{A}_{1}$ and $\mathbf{A}_{2}$ be two disjoint convex sets in the plane with $C^{2}$ boundaries. Then

$$
\kappa=\frac{1}{\sqrt{a^{2}+b^{2}-2 a b \cos (\alpha+\beta)}}\left(\frac{\kappa_{1}}{1+\kappa_{1} a r} b \sin ^{2} \alpha-\frac{\kappa_{2}}{1+\kappa_{2} b r} a \sin ^{2} \beta\right) .
$$

Here $\kappa$ is the curvature in a point $P$ of the conflict set, $\kappa_{i}$ are the curvatures in the feet of $P$ on the boundary of $\mathbf{A}_{i}$, and $r$ is the JM-distance from $P$ to $\mathbf{A}_{i}$; $\alpha$ is the angle between the projection line on $\mathbf{A}_{1}$ and the conflict set at $P$ and $\beta$ similarly with respect to $\mathbf{A}_{2}$.

Proof. We use the kite-construction (cf. Figure 8) in order to determine the normal vector to the conflict set.

We use the notation $\mathbf{n}, \mathbf{n}_{1}, \mathbf{n}_{2}, \mathbf{t}, \mathbf{t}_{1}$ and $\mathbf{t}_{2}$ as before. Let $\alpha$ be the angle between the normal $\mathbf{n}_{1}$ and $\mathbf{t}$ and let $\beta$ be the angle between the normal $\mathbf{n}_{2}$ and $\mathbf{t}$.

We have

where

$$
\mathbf{n}=\frac{b \mathbf{n}_{1}-a \mathbf{n}_{2}}{c} \text { and } \quad \mathbf{t}=-\frac{b \mathbf{t}_{1}+a \mathbf{t}_{2}}{c}
$$

$$
c=\sqrt{a^{2}+b^{2}-2 a b \cos (\alpha+\beta)} .
$$

We replace $\mathbf{A}_{1}$ and $\mathbf{A}_{2}$ by the $r$-JM-distance sets. The boundaries intersect at $P$. This does not influence the conflict set. Also the tangent and normal vectors are not effected.

Let $s$ be arc length for the conflict set and $s_{i}$ for the boundaries of the $r$-distance sets.

$$
\begin{aligned}
\frac{d \mathbf{n}}{d s}=\left(b \frac{d \mathbf{n}_{1}}{d s_{1}} \cdot \frac{d s_{1}}{d s}-a \frac{d \mathbf{n}_{2}}{d s_{2}} \cdot \frac{d s_{2}}{d s}\right) & \frac{1}{c}+\left(b \mathbf{n}_{1}-a \mathbf{n}_{2}\right) \cdot(\ldots) \\
& =\left(b \kappa_{1}^{*} \mathbf{t}_{1} \frac{d s_{1}}{d s}-a \kappa_{2}^{*} \mathbf{t}_{2} \frac{d s_{2}}{d s}\right) \frac{1}{c}+\left(b \mathbf{n}_{1}-a \mathbf{n}_{2}\right) \cdot(\ldots) .
\end{aligned}
$$

In order to compute the curvature $\kappa$ it is now sufficient to take the inner product with $\mathbf{t}$.

Note that

$$
\mathbf{t}_{1} \bullet \mathbf{t}=\sin \alpha ; \quad \mathbf{t}_{2} \bullet \mathbf{t}=\sin \beta ; \quad\left(b \mathbf{n}_{1}-a \mathbf{n}_{2}\right) \bullet \mathbf{t}=0
$$

and moreover

$$
\frac{d s_{1}}{d s}=\sin \alpha ; \quad \frac{d s_{2}}{d s}=\sin \beta
$$

Hence we get

$$
\begin{aligned}
\kappa=\left(b \kappa_{1}^{*} \mathbf{t}_{1} \bullet \mathbf{t} \frac{d s_{1}}{d s}-\right. & \left.a \kappa_{2}^{*} \mathbf{t}_{2} \bullet \mathbf{t} \frac{d s_{2}}{d s}\right) \frac{1}{c} \\
= & \left(b \kappa_{1}^{*} \sin \alpha \frac{d s_{1}}{d s}-a \kappa_{2}^{*} \sin \beta \frac{d s_{2}}{d s}\right) \frac{1}{c}=\frac{b \kappa_{1}^{*} \sin ^{2} \alpha-a \kappa_{2}^{*} \sin ^{2} \beta}{c} .
\end{aligned}
$$

This completes the proof.

N.B. With the above geometric formulas one can replace the expressions in $\alpha$ and $\beta$ by expressions in $\gamma$. Then

$$
\kappa=\frac{b \kappa_{1}^{*}(b-a \cos \gamma)^{2}-a \kappa_{2}^{*}(a-b \cos \gamma)^{2}}{c^{3}} .
$$


EXAMPLE. If the sites are points we get the usual Apolonius situation. The curvature formula reduces after appropriate computations to

$$
\kappa=\frac{b^{2}-a^{2}}{r a b c}=\frac{b^{2}-a^{2}}{a b \cdot d(A, B)} .
$$

This is in harmony with the formula for the radius of the Appolonius circle

$$
R=\frac{a b}{b^{2}-a^{2}} \cdot d(A, B)
$$

5. The distance foliation. For any point in the plane, we can consider the ratio $d(x, \mathbf{A}): d(x, \mathbf{B})$. This defines a function from the Euclidean plane $\mathcal{E}$ to $[0, \infty]$. The level lines foliate the plane and they are just the JM-conflict lines for the given weight $a: b$, unless $a=0$ or $b=0$ (cf. Figure 11). The tangent lines to the foliation are given by the 'kite'-construction.

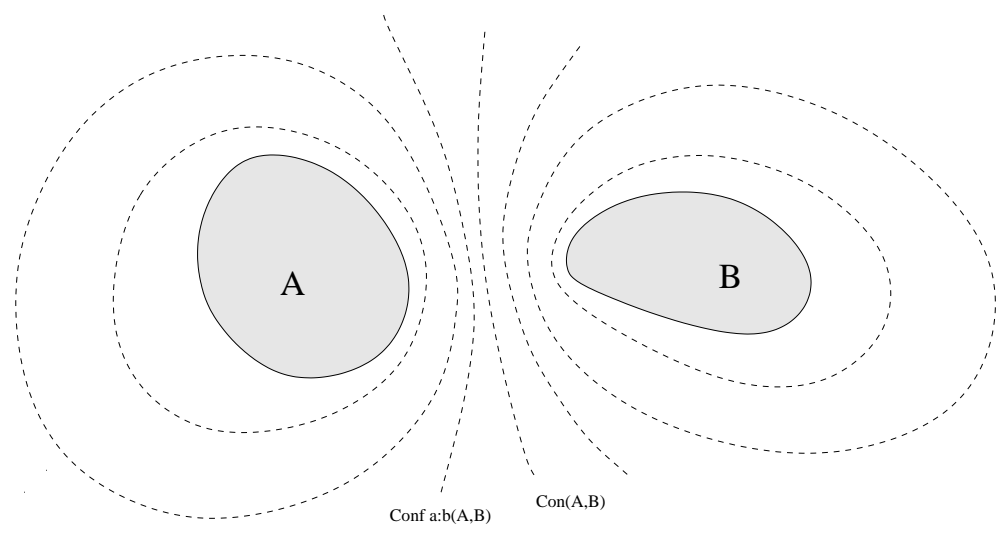

Figure 11. The distance foliation

\section{References}

[Be] M. Berger, Geometry I and II, Universitext, Springer, Berlin, 1987.

[BG] J. W. Bruce, P. J. Giblin, Curves and Singularities, Cambridge Univ. Press, Cambridge, 1984.

[Cox] H. S. M. Coxeter, Introduction to Geometry, Wiley, New York, 1961.

[Go] A. Goddijn, Smoothness Properties of Conflict Sets (in Dutch), Nascholingscursus Achtergronden van de Meetkunde, Utrecht, 1997 (translation in preparation).

[Ma] M. van Manen, Thesis Project on Conflict Sets (in progress), Utrecht University.

[Mi] J. Milnor, Morse Theory, Ann. of Math. Stud. 51, Princeton Univ. Press, Princeton, 1963.

[Ok] A. Okabe, B. Boots, K. Sugihara, Spatial Tesselations: Concepts and Applications of Voronoi Diagrams, Wiley Ser. Probab. Math. Statist. Appl. Probab. Statist., Wiley, Chichester, 1992.

[Po] I. R. Porteous, Geometric Differentiation for the Intelligence of Curves and Surfaces, Cambridge Univ. Press, Cambridge, 1994.

[SSG] J. Sotomayor, D. Siersma, R. Garcia, Curvatures of conflict surfaces in Euclidean 3-space, this volume. 